\title{
AMENDMENTS
}

\section{Publisher Correction: Evolution of chalcone isomerase from a noncatalytic ancestor}

Miriam Kaltenbach, Jason R. Burke, Mirco Dindo, Anna Pabis, Fabian S. Munsberg, Avigayel Rabin, Shina C. L. Kamerlin (D, Joseph P. Noel (iD and Dan S. Tawfik

Correction to https://doi.org/10.1038/s41589-018-0042-3, published online 23 April 2018

In the version of this article originally published, the number for the equal contributions footnote was missing for Miriam Kaltenbach and Jason R. Burke in the author list. The error has been corrected in the PDF and print versions of this article.

\section{Publisher Correction: The Jumonji-C oxygenase JMJD7 catalyzes (3S)-lysyl hydroxylation of TRAFAC GTPases}

Suzana Markolovic, Qinqin Zhuang, Sarah E. Wilkins, Charlotte D. Eaton, Martine I. Abboud (D), Maximiliano J. Katz, Helen E. McNeil, Robert K. Leśniak, Charlotte Hall, Weston B. Struwe, Rebecca Konietzny, Simon Davis (D), Ming Yang, Wei Ge (D, Justin L. P. Benesch (D), Benedikt M. Kessler (D), Peter J. Ratcliffe (D), Matthew E. Cockman (D), Roman Fischer (D), Pablo Wappner, Rasheduzzaman Chowdhury, Mathew L. Coleman and Christopher J. Schofield

Correction to: Nature Chemical Biology https://doi.org/10.1038/s41589-018-0071-y, published online 18 June 2018.

In the version of this article initially published, authors Sarah E. Wilkins, Charlotte D. Eaton, Martine I. Abboud and Maximiliano J. Katz were incorrectly included in the equal contributions footnote in the affiliations list. Footnote number seven linking to the equal contributions statement should be present only for Suzana Markolovic and Qinqin Zhuang, and the statement should read "These authors contributed equally: Suzana Markolovic, Qinqin Zhuang." The error has been corrected in the HTML and PDF versions of the article. 